\title{
EFFECT OF VOXEL SIZE ON THE ACCURACY OF NERVE TRACING MODULE OF CONE BEAM COMPUTED TOMOGRAPHY IMAGES
}

\author{
Mostafa S. Ashmawy*, Ashraf A. Abou-Khalaf ${ }^{* *}$ and Raghdaa A. Mostafa***
}

\begin{abstract}
Introduction: Cone beam computed tomography (CBCT) has been used for preoperative treatment planning for dental implants, one of the major advantages is its accurate linear measurements. But when it comes to automatic detection of voxel intensity values as in automatic nerve detection, little evidence in the literature was found. Thus; in order to examine the accuracy of this automatic process, this study was performed in vitro.

Methods: A dry edentulous mandible was imaged using i-CAT next generation (Imaging sciences international, Hatfield, PA, USA) using gutta percha markers at certain areas of interest, the reconstructed panoramic image was evaluated and nerve tracing was done for both sides, then linear measurements was performed from the edge of the inferior alveolar nerve canal (IANC) to the inferior border of mandible, buccal, lingual and crest of the ridge. These measurements were compared to actual physical measurements performed by using a digital caliber after sectioning of the mandible at the gutta percha sites.
\end{abstract}

Results: Intra-observer agreement was good to very good regarding all measurements for both observers, while inter observer agreement was weak to very good regarding all measurements for both observers. There was no statistically significant difference between the CBCT scans $(0.2 \mathrm{~mm}$, $0.3 \mathrm{~mm}$, and $0.4 \mathrm{~mm}$ voxel size) regarding all measurements except one measurement at the $0.4 \mathrm{~mm}$ voxel size CBCT scan showed a statistically significant high mean error.

Conclusion: The choice of FOV and voxel size should be made by clinicians based on the clinical task at hand, keeping in mind that their choices not only affect the diagnostic quality of images but also the amount of radiation exposure that their patients receive.

\section{INTRODUCTION}

Dental implants have evolved over the years and turned into a predictable treatment for replacement of missing teeth..$^{[1,2]}$ Precise preoperative planning and assessment of the recipient site is essential for a successful implant osseointegration and successful treatment outcome. ${ }^{[3]}$ This requires a comprehensive knowledge of various anatomic structures and variations..$^{[4]}$

\footnotetext{
* Lecturer of Oral Radiology, Oral Radiology Dept. Faculty of Dentistry, Ain-Shams University.

** Professor of Oral Radiology and Diagnostic Science, Oral Radiology Dept., Faculty of Dentistry, King Abdulaziz

University and Ain-Shams University.

**** Lecturer of Oral Radiology, Oral Radiology Dept. Faculty of Dentistry, Ain-Shams University
} 
While the number of dental implants have increased, the numbers of neurosensory disturbances and haemorrhages have increased even in locations which was considered safe before as the anterior mandible. ${ }^{[5,6]}$ Therefore, preoperative planning must be done to identify the inferior alveolar nerve canal (IANC) to prevent damage to the neurovascular bundle that passes inside the mandibular canal, the course of which varies within the mandibular body as well as the shape and dimensions of the bone. ${ }^{[7]}$

Several authors investigated the mandibular canal position using cone beam computed tomography (CBCT), IANC proved to run near the lingual cortical side from its entrance in the ramus to the mental foramen, and that it is positioned about $1 \mathrm{~cm}$ above the inferior border of the mandible. ${ }^{[8-10]}$ On the other hand other researches found that IANC position may vary from one patient to the other according to the degree of alveolar ridge resorption. ${ }^{[10,11]}$

Although CBCT has gained increased popularity in many indications, its main indication is dental implant procedures ${ }^{[12]}$, specially providing a superior display of the IANC and its variations which is critical to perform a more expected surgical procedures, avoiding possible sensory disturbances and complications. $^{[13]}$

Viewing CBCT images using the software viewer is considered essential during implant planning either by the oral radiologist or the oral surgeon. All CBCT systems have their viewing software supplied on a compact disk (CD); each software is different in its task specific reconstruction capabilities provided by the manufacturer. Although these features may include panoramic reconstructions, implant planning reconstructions with 2-dimensional (2D) and 3- dimensional (3D) windows, temporomandibular joint reconstructions, airway reconstructions. Almost any CBCT software has a nerve tracing application. This allows for the identification and colour coding of IANC, thereby assisting its recognition. ${ }^{[4,15]}$
Up to our knowledge, few researches were focusing on the accuracy of the nerve tracing tool in order to provide sufficient scientific information for the dental community, so the aim of the current study is to determine the accuracy of using IANC tracing technique on CBCT images in precisely locating the IANC using different voxel sizes.

\section{MATERIALS AND METHODS}

For the purpose of our study, one edentulous mandible, randomly selected, was borrowed from the Anatomy Department, Faculty of Medicine, Ain Shams University. The study design was expedited from review by the Faculty's Research Ethics Committee. The mandible was anonymous and not identified by age, gender, or ethnic group.

\section{Mandible preparation:}

The alveolar crest of the mandible in both sides at the molar, premolar areas was plateaued using a diamond bur mounted on straight hand piece. The buccal and lingual cortical plates came to be nearly on the same horizontal level in order to facilitate reference points determination.

\section{Creating reference points:}

In order to create reference markers in the mandible, small pieces of gutta percha were fixed on the mandible with glue in a five predefined locations to yield 5 dentoalveolar specimens (Fig. 1). The position of the reference markers were at the level of the crestal bone between the sockets of the premolar and molar teeth. Other reference markers were positioned along a perpendicular line drawn to the inferior border of the mandible from the previous reference markers at each location. This was done to ensure reproducible measurements. Individual sections were assigned a numeric value of 1 through 5 , starting anteriorly in a posterior direction. $\mathrm{R}$ and $\mathrm{L}$ letters were used to discriminate the two sides. 


\section{Radiographic Scanning:}

The mandible was wrapped with thin plastic wrapping (in order not to disturb the dryness of the mandible) and placed in a thin, clear plastic container which was filled with water to simulate soft tissue attenuation. Then, the mandible was scanned using the i-CAT next generation (Imaging Sciences International, Hatfield, PA, USA) at the Oral Radiology Department, Faculty of dentistry, Ain shams university. (Fig. 2)

The assembly was adjusted on the machine's platform in a central position which was checked with the three laser beam. Images were obtained at 3 different voxel sizes $0.2 \mathrm{~mm}, 0.3 \mathrm{~mm}$ and $0.4 \mathrm{~mm}$. All the three scans were performed at $120 \mathrm{kVp}, 5$ $\mathrm{mA}$. The field of view (FOV) was adjusted in order to cover the sigmoid notch superiorly and the mandibular inferior border inferiorly in order to easily locate the mandibular foramen and inferior mandibular border respectively on the resultant image.

\section{Nerve tracing and radiographic measurements}

After volume acquisition, images were examined by two oral and maxillofacial (OMF) radiologists with ten and fourteen years of experience. Images were examined using i-CAT Vision ${ }^{\circledR}$ software (Imaging Sciences International, Hatfield, PA, USA). The examiners separately reviewed the volumes in ideal dimly lit viewing conditions using the same computer monitor (15.6 inch HD LED) at full size, 1:1. They were allowed to handle the volumes freely with the software and use all available tools to identify the IANC. They were also allowed to change contrast and brightness.

\section{IANC tracing and measurements:}

On the implant screen, panoramic reconstruction was centrally adjusted along an intermediate level between the crest of the ridge and mandibular inferior border. Manual IANC tracing was performed using the "estimate nerve canal" tool provided by the software; right and left IANCs were traced separately based on four points starting from the mandibular foramen down to the mental foramen

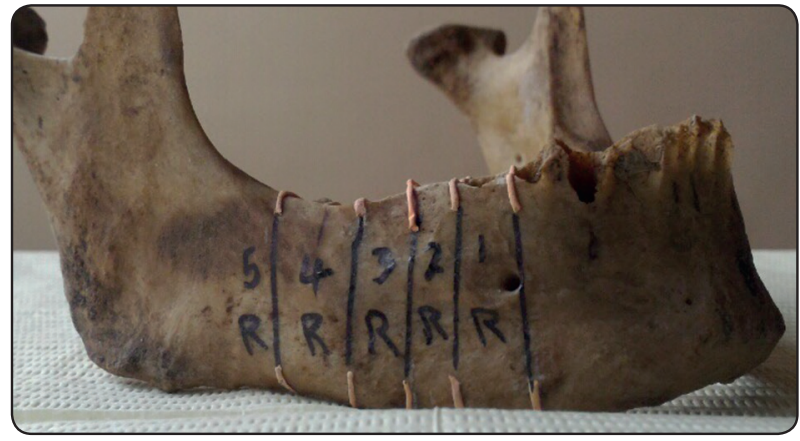

Fig. (1) Gutta percha markers fixed on the dry mandible

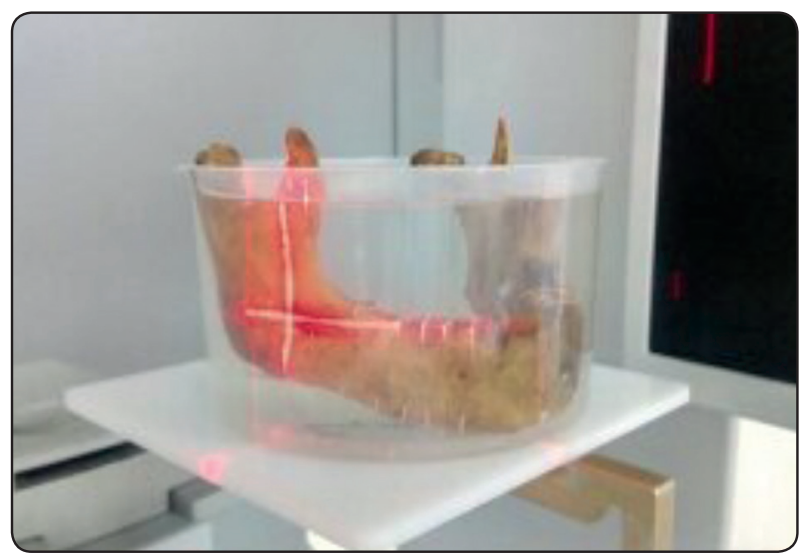

Fig. (2) Positioning of the mandible on the platform during CBCT acquisition

as displayed on cross-sectional images. The border of the color coded tracing was used as the starting measurement point as shown in (Fig. 3)

Then cross sectional images were reoriented in order to pass through gutta percha markers in order to precisely standardize measurements' locations. On each section passing through gutta percha markings, four linear measurements were performed using the "distance" tool. The distance from IANC superior, inferior, buccal and lingual edges to the alveolar crest, inferior, buccal and lingual mandibular borders respectively were measured at each section using a perpendicular line as shown in (Fig. 3). Each observer performed the measurements twice separated by two weeks interval using the same protocol. 


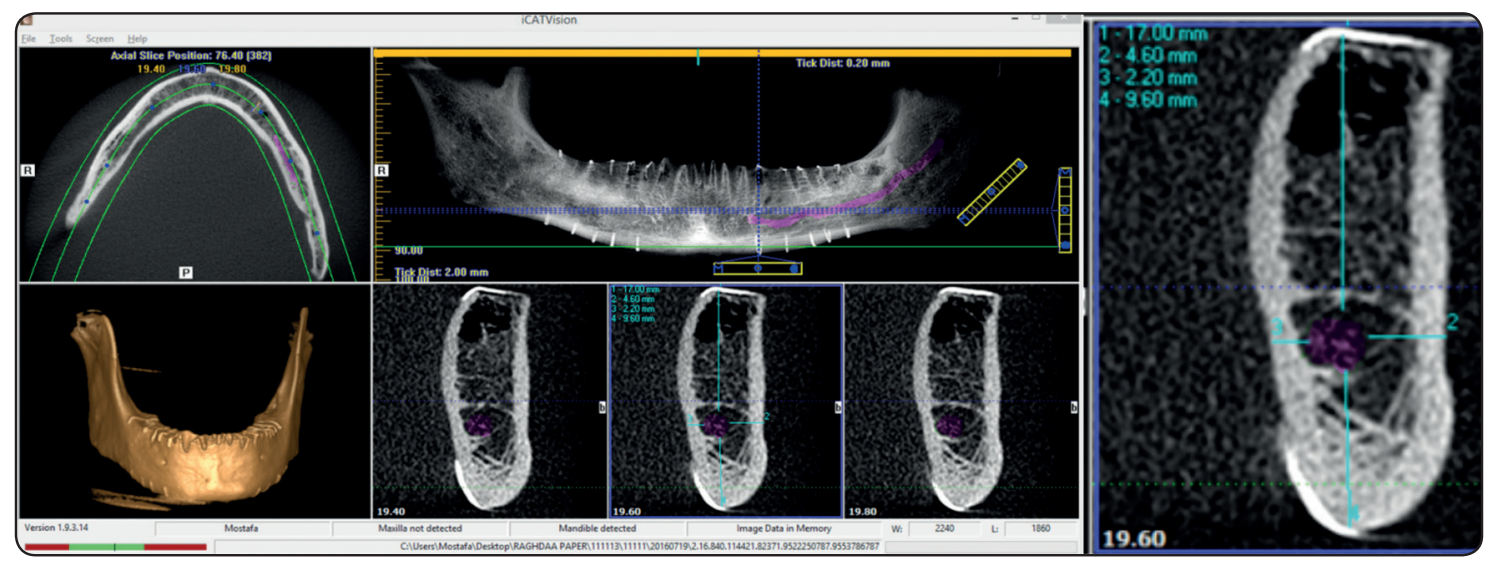

Fig. (3) Implant screen on i-CAT VisionR showing the color coded nerve tracing on both cross sectional and panoramic images

\section{Physical Measurements}

After scanning, the dry mandible was sectioned using a Stryker saw (Stryker Model 810 Autopsy Saw, Azusa, CA) at the 5 predefined locations along the lines joining the reference markers from the alveolar crest to the inferior boarder of the mandible on each side as shown in (Fig. 4). For each section, the distance from the IANC border to the alveolar crest, inferior, buccal and lingual mandibular borders was measured by the caliber using the gutta percha markers as a guide for measurement. These measurements were recorded for further comparison with CBCT measurements.

\section{Statistical Analysis}

Numerical data were explored for normality by checking the distribution of data and using tests of normality (Kolmogorov-Smirnov and ShapiroWilk tests). Measurement error data showed nonparametric distribution. Data were presented as mean, median, standard deviation (SD), minimum, maximum and 95\% Confidence Interval (95\% CI) values. Intra- and inter-observer agreement was assessed using Cronbach's alpha reliability coefficient. Friedman's test was used to compare between measurement error with the three CBCT scans $(0.2$, 0.3 , and $0.4 \mathrm{~mm}$ voxel size). Wilcoxon signed-rank test with Bonferroni's adjustment was used for pair-wise comparisons when Friedman's test is significant. The significance level was set at $\mathrm{P} \leq 0.05$. Statistical analysis was performed with IBM SPSS Statistics Version 20 for Windows (SPSS, Inc., an IBM Company, NY, USA).

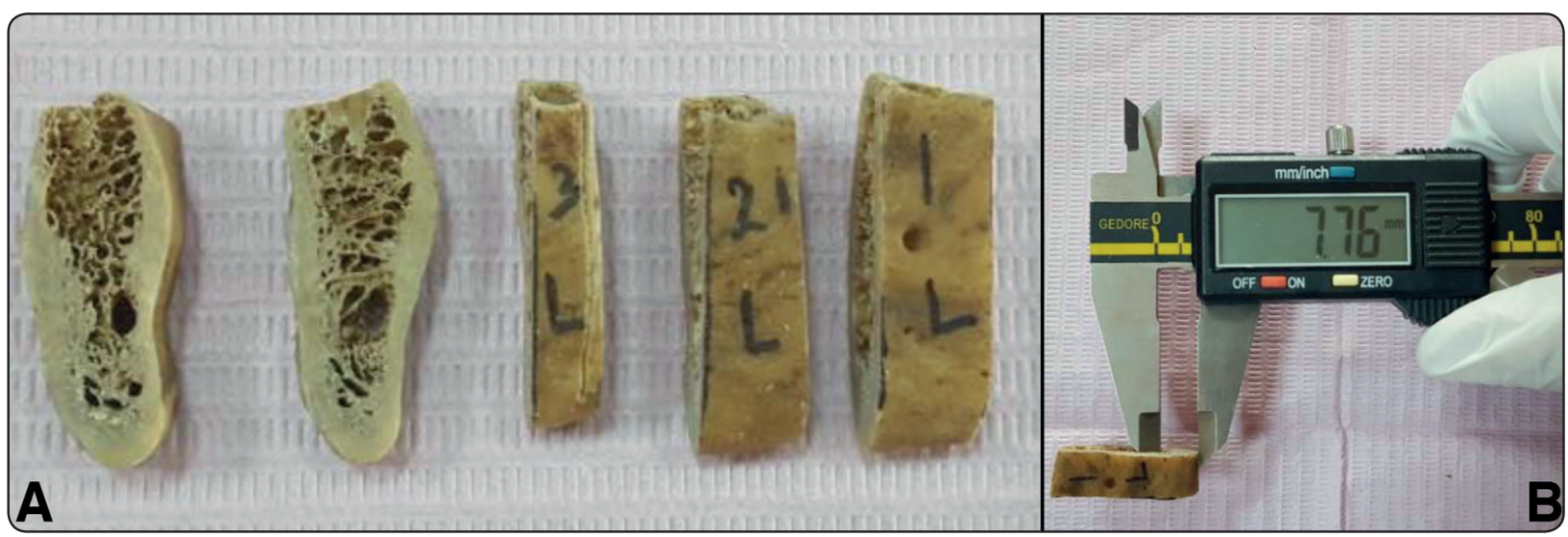

Fig. (4): A) Dento-alveolar specimens after cutting and B) Physical measurement of the mandibular sections 


\section{RESULTS}

\section{Reliability analysis}

As the two observers performed the measurements twice using the same protocol, both showed good to very good intra-observer agreement regarding all measurements with Cronbach's alpha values ranging from $0.685-0.994$ and $0.764-0.999$ for the first and second observers respectively. Comparing both readings by both observers showed weak to very good inter-observer agreement regarding all measurements with Cronbach's alpha values ranging from $0.074-0.985$ and $0.066-0.990$ for the first and second readings respectively.

\section{Error measurement}

The difference between the actual physical measurements obtained by the digital caliber and $\mathrm{CBCT}$ measurements was considered as the measurement error and by comparing this error between the CBCT scans $(0.2 \mathrm{~mm}, 0.3 \mathrm{~mm}$, and 0.4 $\mathrm{mm}$ voxel size), there was no statistically significant difference between these CBCT scans regarding all measurements (Fig. 5) except one measurement at the $0.4 \mathrm{~mm}$ voxel size CBCT scan showed a statistically significant high mean error; while all other measurements showed no statistically significant difference between the CBCT measurements and actual physical measurements as shown in Table (1).

TABLE (1) The mean, standard deviation (SD) values and results of Friedman's test and Wilcoxon signedrank test for comparison between error measurements with different Voxel sizes

\begin{tabular}{|c|c|c|c|c|c|c|c|c|}
\hline \multirow{2}{*}{ Side } & \multirow{2}{*}{ Distance Measured } & \multicolumn{2}{|c|}{$0.2 \mathrm{~mm}$ Voxel } & \multicolumn{2}{|c|}{$0.3 \mathrm{~mm}$ Voxel } & \multicolumn{2}{|c|}{$0.4 \mathrm{~mm}$ Voxel } & \multirow{2}{*}{$P$-value } \\
\hline & & Mean & SD & Mean & SD & Mean & SD & \\
\hline \multirow{4}{*}{$\begin{array}{c}\text { Left } \\
\text { IANC }\end{array}$} & IANC/Alveolar crest & 0.69 & 0.75 & 0.67 & 0.98 & -0.11 & 0.32 & 0.071 \\
\hline & IANC/Inferior border & 1.15 & 1.66 & 0.73 & 0.65 & 0.87 & 1.02 & 0.779 \\
\hline & IANC/Buccal border & 0.56 & 1.49 & -0.40 & 1.15 & -0.75 & 0.86 & 0.127 \\
\hline & IANC/Lingual border & -0.20 & 0.29 & 0.00 & 0.45 & 0.28 & 0.47 & 0.607 \\
\hline \multirow{4}{*}{$\begin{array}{l}\text { Right } \\
\text { IANC }\end{array}$} & IANC/Alveolar crest & 1.46 & 1.89 & 2.14 & 2.78 & 0.49 & 0.98 & 0.779 \\
\hline & IANC/Inferior border & $-0.65^{\text {в }}$ & 1.86 & $-0.81^{\text {в }}$ & 3.01 & $1.55^{\mathrm{A}}$ & 1.41 & $0.022 *$ \\
\hline & IANC/Buccal border & -0.25 & 0.49 & 0.52 & 0.47 & 0.16 & 1.74 & 0.165 \\
\hline & IANC/Lingual border & -0.49 & 0.28 & -0.53 & 0.63 & -0.68 & 0.39 & 0.331 \\
\hline
\end{tabular}

*: Significant at $P \leq 0.05$, Different superscripts in the same row are statistically 


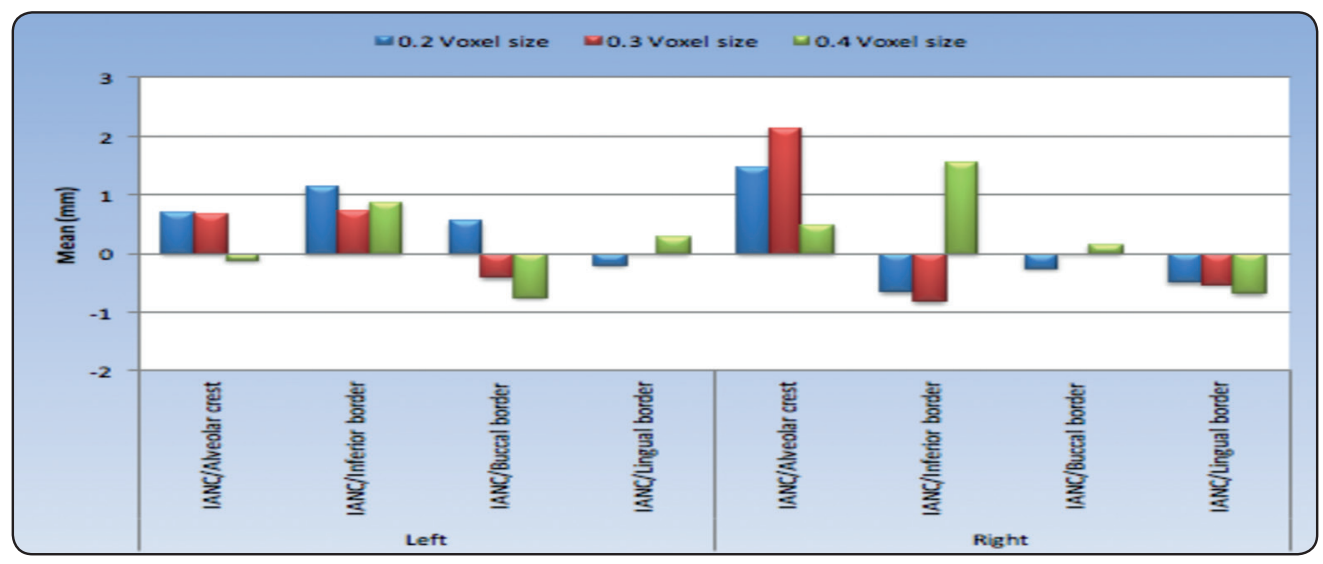

Fig. (5) Bar chart representing comparison between mean error measurements with different Voxel sizes

\section{DISCUSSION}

IANC is one of the most important anatomical structures in the mandible taking into consideration the variation in its course in both vertical and horizontal planes ${ }^{[4]}$. Moreover, the visibility of the canal varies between patients and in different locations in the mandible being more easily identified in posterior areas and more difficult towards the mental foramen ${ }^{[13]}$. Therefore, precise identification of the IANC for accurate preoperative planning is a challenging process ${ }^{[16]}$.

This points out the precious contribution of $3 \mathrm{D}$ CBCT technology as an ideal imaging modality owed to its sub-millimeter resolution and high image quality which allows mandibular canal tracing to determine its exact location ${ }^{[16,17]}$.

Clinically, the quality of the CBCT image and its capability to demonstrate the anatomical structures and pathology depend on settings used during acquisition or reconstruction, one influencing factor is the voxel size which has a huge impact on the image details and the diagnostic outcome ${ }^{[18]}$.

Therefore, the aim of this study was to investigate the impact of using three different voxel sizes in detecting the accuracy of IANC tracing in vitro. To the authors' knowledge, no published studies assessed the use of isolated voxel size variation in
CBCT images on the visualization of IANC specially by using the nerve tracing tool. Although other authors investigated the effect of changing the voxel size on detection of periapical bony lesions ${ }^{[19]}$, detection of external root resorption ${ }^{[20]}$, accuracy of linear measurements ${ }^{[21-23]}$, accuracy of $3 \mathrm{D}$ reconstruction $^{[24]}$, accuracy of mandibular cortical thickness measurements ${ }^{[25]}$ and temporomandibular joint osseous changes ${ }^{[26]}$.

In the present study we used a dry mandible for testing CBCT linear measurements by comparing these measurements with the actual physical measurements as the gold standard. The imaging of a dry mandible is a challenge regarding proper positioning and choosing the best FOV. Also we have chosen to immerse the mandible in water to simulate soft tissues during image acquisition. This was in accordance with several authors who imaged a dry mandible for different purposes regarding

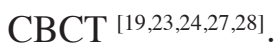

We have chosen to use gutta percha markers to reorient the $\mathrm{CBCT}$ cross sectional images and to identify measurement areas in order to accurately standardize measurement areas; Gutta percha is widely used as a radiographic marker in several CBCT studies ${ }^{[29,30,31]}$; on the other hand Damstra et $\mathrm{al}^{[23]}$ used glass sphere markers, Hekmatian et al ${ }^{[25]}$ 
used aluminum foil strips and Ludlow et a ${ }^{[27]}$ used orthodontic wires in their studies. In our study we have chosen gutta percha to avoid possible metal artefacts which may result from metallic markers that may affect image quality.

In our study we compared the effect of changing the voxel size during image acquisition on the accuracy of nerve tracing and we found that there was no statistically significant difference between $0.2,0.3$ and $0.4 \mathrm{~mm}$ voxel sizes.

The same results were proved by Aktan et $\mathrm{al}^{[21]}$ and sherrard et al. ${ }^{[32]}$ who compared accuracy of endodontic working length by using different voxel sizes ranging from 0.1 to $0.5 \mathrm{~mm}$ and 0.2 to 0.4 respectively, they concluded that there is no statistically significant difference between the CBCT scans regarding endodontic working length measurement.

Damstra et $\mathrm{al}^{[23]}$ and Vieira et $\mathrm{al}^{[33]}$ showed the same results regarding the accuracy of CBCT linear measurements when they compared $0.25,0.4 \mathrm{~mm}$ and $0.125,0.25 \mathrm{~mm}$ voxel sizes respectively. The same results were obtained by Hekmatian et al ${ }^{[25]}$ regarding mandibular thickness measurements as they found no significant difference between 0.15 and $0.3 \mathrm{~mm}$ voxel sizes. Our results were also in accordance with Lukat et a ${ }^{[26]}$ who investigated the visualization of TMJ osseous structures by 0.076 and 0.3 voxel sizes and Ozer ${ }^{[34]}$ who compared $0.125,0.2,0.3$ and $0.4 \mathrm{~mm}$ voxel size in detection of simulated vertical root fracture.

Despite that all our measurements showed no statistically significant mean error, only one measurement showed statistically significant mean error and this could possibly be due to bone marrow spaces present at this specific area (right side from the inferior border of IANC to the mandibular inferior border) causing difficulty for the software to accurately determine the exact border of IANC at that area; as the software identifies the IANC by searching for high-contrast gaps in the mandible, so similarity between the voxel value of IANC and a large marrow space (probably larger than $0.4 \mathrm{~mm}$ ) might be the cause of this difference in mean error.

While our results showed no effect on the image accuracy when changing the voxel size, we have to stress on the fact that our study focused on linear measurements while other authors investigated the accuracy of 3D volumetric measurements and showed different results as Maret et al ${ }^{[24]}$ who compared between 0.2 and $0.3 \mathrm{~mm}$ voxel size in 3D volumetric measurements and found a statistically significant underestimation of the volume in 0.3 mm CBCT images. Also Dalili et al. ${ }^{[35]}$ concluded in their study regarding detection of external root resorption that $0.25 \mathrm{~mm}$ was more accurate than 0.5 $\mathrm{mm}$ voxel size.

The increased demand on CBCT for implant planning and the convenient tools provided by various software, increased dentists' referral for CBCT scans. This should be an alarming sign for oral radiologists to investigate the effect of increased patient absorbed dose and provide recommendations for patients exposure to radiation. Several parameters can be varied during image acquisition according to the diagnostic task but still no protocols have been established for specific diagnostic tasks in dentistry ${ }^{[18]}$. In our study we proved that changing the voxel size will not affect the accuracy of the nerve tracing tool, and so using larger voxel size will not affect the clinical outcome and decrease the patient absorbed dose according to Nikneshan et $\mathrm{al}^{[36]}$.

This would be following the ALARA concept and in accordance with the American academy of oral and maxillofacial radiology (AAOMR) $)^{[37]}$ and the European academy of dental and maxillofacial radiology (EADMFR) ${ }^{[38]}$ as both stated that when using CBCT, the smallest field of view should be used in order to decrease patient absorbed dose.

On the other hand, using a larger voxel size will decrease the scanning time, thus decreasing motion artefacts which may cause a dramatic effect on image quality ${ }^{[39]}$. 


\section{CONCLUSION}

The choice of voxel size should be made by clinicians based on the clinical task at hand, keeping in mind that their choices not only affect the diagnostic quality of images but also the amount of radiation exposure that their patients receive.

\section{RECOMMENDATIONS}

The objectives of this study did not include the assessment of diagnostic accuracy, the performance of the imaging software, or the resolution of the computer monitor. Future studies should be performed assessing the diagnostic accuracy of CBCT Images using varying imaging protocols. So further clinical studies in this area are needed in order to examine the accuracy of nerve tracing tools.

\section{Conflict of Interests}

The authors declare that there is no conflict of interests regarding the publication of this paper.

\section{REFERENCES}

1. Henry PJ,Laney WR, Jemt T, Osseointegrated implants for single-tooth replacement: a prospective 5-year multicenter study. Int J Oral Maxillofac Implants. 1996;11:450-455.

2. Li B, Wang Y, Li J. A feasibility study of applying conebeam computed tomography to observe dimensional changes in human alveolar bone. J Zhejiang Univ Sci B. 2014 Apr;15(4):393-8.

3. Jacobs R, Van Steenberghe D . Radiographic Planning and Assessment of Endosseous Oral Implants. Berlin, Germany: Springer-Verlag; 1998.

4. Wang HL, Juodzbalys G. Guidelines for the Identification of the Mandibular Vital Structures: Practical Clinical Applications of Anatomy and Radiological Examination Methods. Journal of Oral \& Maxillofacial Research. 2010;1(2)e1

5. Mraiwa N, Jacobs R, Van Clyeynenbreugel J. The nasopalatine canal revisited using 2D and 3D CT imaging. Dentomaxillofac Radiol. 2004;33:396-402.

6. Kieser J , Kuzmanovic D , Payne A, Dennison J , Herbison $P$. Patterns of emergence of the human mental nerve. Arch Oral Biol. 2002;47:743-747. [CrossRef] [Medline]
7. Juodzbalys G, Wang HL, Sabalys G . Anatomy of mandibular vital structures. Part II: mandibular incisive canal, mental foramen and associated neurovascular bundles in relation with dental implantology. J Oral Maxillofac Res. 2010;1:e3

8. Gerlach NL, Meijer GJ, Kroon DJ, Bronkhorst EM, Bergé SJ, Maal TJ. Evaluation of the potential of automatic segmentation of the mandibular canal using cone-beam computed tomography. Br J Oral Maxillofac Surg. 2014 Nov;52(9):838-44.

9. Kwon KH, Sim KB, Lee JM. Evaluation of the course of the inferior alveolar canal in the mandibular ramus using cone beam computed tomography. Korean Assoc Oral Maxillofac Surg 2012;38:231-9.

10. Li N, Zhao B, Tan C. Intramandibular course and anatomic structure of the inferior alveolar nerve canal. Zhonghua Kou Qiang Yi Xue Za Zhi 2001;36:446-7.

11. Jui-Ting Hsu, Heng-Li Huang, Lih-Jyh Fuh, Rou-Wei Li, Jay Wu, Ming-Tzu Tsai, Yen-Wen Shen, and MingGene Tu. Location of the Mandibular Canal and Thickness of the Occlusal Cortical Bone at Dental Implant Sites in the Lower Second Premolar and First Molar. Computational and Mathematical Methods in Medicine Volume 2013 Article ID 608570, 8 pages.

12. Poeschl PW, Schmidt N, Guevara-Rojas G, Seemann R, Ewers R, Zipko HT, Schicho K. Comparison of cone-beam and conventional multislice computed tomography for image-guided dental implant planning. Clin Oral Investig. 2013 Jan;17(1):317-24

13. De Oliveira-Santos C, Souza PH, de Azambuja BertiCouto S, Stinkens L, Moyaert K, Rubira-Bullen IR, Jacobs R. Assessment of variations of the mandibular canal through cone beam computed tomography. Clin Oral Investig. 2012 Apr;16(2):387-93

14. Prashant P Jaju and Sushma P Jaju. Clinical utility of dental cone-beam computed tomography: current perspectives. Dove press journal. 2014 Apr;6:29-43.

15. Kenneth Abramovich and Dwight D. Rice. Basic Principles of Cone Beam Computed Tomography. Dental clinic journal.2014 Jul;58(3):463-484.

16. Gerlach NL, Meijer GJ, Maal TJ, Mulder J, Rangel FA, Borstlap WA, Bergé SJ. Reproducibility of 3 different tracing methods based on cone beam computed tomography in determining the anatomical position of the mandibular canal. J Oral Maxillofac Surg. 2010 Apr;68(4):811-7. 
17. Saati S, Kaveh F, Yarmohammadi S. Comparison of Cone Beam Computed Tomography and Multi Slice Computed Tomography Image Quality of Human Dried Mandible using 10 Anatomical Landmarks. J Clin Diagn Res. 2017 Feb;11(2):ZC13-ZC16.

18. Rubens Spin-Neto, Erik Gotfredsen, and Ann Wenzel. Impact of Voxel Size Variation on CBCT-Based Diagnostic Outcome in Dentistry: a Systematic Review. J Digit Imaging. 2013 Aug; 26(4): 813-820.

19. Shirin Sakhdari, Ahmad Reza Talaeipour, Maziar Talaeipour, Maryam Pazhutan, Sanaz Heidarkhan Tehrani and Mohammad Javad Kharazifard. Diagnostic Accuracy of CBCT with Different Voxel Sizes and Intraoral Digital Radiography for Detection of Periapical Bone Lesions: An Ex-Vivo Study. J Dent (Tehran). 2016 Mar; 13(2): 77-84.

20. Gabriela Salatino Liedke, Heloísa Emília Dias da Silveira, Heraldo Luis Dias da Silveira, Vinícius Dutra and José Antônio Poli de Figueiredo. Influence of Voxel Size in the Diagnostic Ability of Cone Beam Tomography to Evaluate Simulated External root resorption. Journal of Endodontics;2009 Nov;35(2):233-235.

21. Ali Murat Aktan, Cihan Yildirim, Emrah Karataşlıŏlu, Mehmet Ertuğrul Çiftçi, Fatih Aksoy. Effects of Voxel Size and Resolution on the Accuracy of Endodontic Length Measurement Using Cone Beam Computed Tomography. Annals of anatomy J. 2016;208:96-102.

22. Patcas R, Müller L, Ullrich O, Peltomäki T. Accuracy of cone-beam computed tomography at different resolutions assessed on the bony covering of the mandibular anterior teeth. Am J Orthod Dentofacial Orthop. 2012 Jan;141(1):41-50.

23. Damstra J, Fourie Z, Huddleston Slater JJ, Ren Y. Accuracy of linear measurements from cone-beam computed tomography-derived surface models of different voxel sizes. Am J Orthod Dentofacial Orthop. 2010 Jan;137(1):16.e1-6; discussion 16-7.

24. D Maret, N Telmon, O A Peters, B Lepage, J Treil, J M Inglèse, A Peyre, J L Kahn and M Sixou. Effect of voxel size on the accuracy of 3D reconstructions with cone beam CT. Dentomaxillofac Radiol. 2012 Dec; 41(8): 649-655.

25. Ehsan Hekmatian, Nasim Jafari-Pozve and Ladan Khorrami. The effect of voxel size on the measurement of mandibular thickness in cone-beam computed tomography. Dent Res J (Isfahan). 2014 Sep-Oct; 11(5): 544-548.

26. Lukat TD, Perschbacher SE, Pharoah MJ, Lam EW. The effects of voxel size on cone beam computed tomography images of the temporomandibular joints. Oral Surg Oral Med Oral Pathol Oral Radiol. 2015 Feb;119(2):229-37.

27. John B. Ludlow, William Stewart Laster, L'Tanya J. Bailey and H. Garland Hershey. Accuracy of measurements of mandibular anatomy in cone beam computed tomography images. Oral Surg Oral Med Oral Pathol Oral Radiol Endod. 2007 Apr; 103(4): 534-542.

28. Marco A. Albuquerque, Bruno F. Gaia and Marcelo GP Cavalcanti. Comparison between multislice and cone-beam computerised tomography in the volumetric assessment of cleft palate. Oral Surgery, Oral Medicine, Oral Pathology, Oral Radiology, and Endodontology, Aug 2011;112(2):249-257.

29. Isabela M. Silva, Deborah Q. Freitas, Glaucia M. Ambrosano, Frab N. Boscolo and Solange M. Almeida. Bone density: comparative evaluation of Hounsfield units in multislice and cone-beam computed tomography. Brazilian Oral Research, 2012 Dec;26(6):550-556.

30. Frederico S. Neves, Taruska V. Vasconcelos, Anne C. Oenning, Sergio L. de-Azevedo-Vaz, Solange M. de Almeida, Deborah Q. Freitas. Oblique or orthoradial CBCT slices for preoperative implant planning: which one is more accurate?. Brazilian Journal of Oral Science, 2014 Jun;13(2):104-108.

31. AA Al-Ekrish and M Ekram. A comparative study of the accuracy and reliability of multidetector computed tomography and cone beam computed tomography in the assessment of dental implant site dimensions. Dentomaxillofac Radiol. 2011 Feb; 40(2): 67-75.

32. Sherrard JF, Rossouw PE, Benson BW, Carrillo R and Buschang PH. Accuracy and reliability of tooth and root lengths measured on cone-beam computed tomographs. Am J Orthod Dentofacial Orthop. 2010 Apr;137(4 Suppl): S100-8.

33. Raiza M. Vieira, Karina C. P. Santos, Denise S. Haddad, Claudio Costa, Jefferson X. Oliveira. Influence of Voxel Size on the Accuracy of Linear Measurements Taken in CBCT Images. Clin Lab Res Den 2014;20(4): 228-33.

34. Senem Yigit Ozer. Detection of Vertical Root Fractures by Using Cone Beam Computed Tomography with Variable Voxel Sizes in an In Vitro Model. Journal of Endodontics. 2011 Jan;37(1):75-79.

35. Dalili Z, Taramsari M, Mousavi Mehr SZ, Salamat F. Diagnostic value of two modes of cone-beam computed tomography in evaluation of simulated external root 
resorption: an in vitro study. Imaging Sci Dent. 2012 Mar;42(1):19-24.

36. Sima Nikneshan, Mahmood R. Aghamiri, Ehsan Moudi, Nika Bahemmat and Hoora Hadian. Dosimetry of Three Cone Beam Computerized Tomography Scanners at Different Fields of View in Terms of Various Head and Neck Organs. Iran J Radiol. 2016 July; 13(3):e34220.

37. Tyndall DA, Price JB, Tetradis S, Ganz SD, Hildebolt C, Scarfe WC. Position statement of the American Academy of Oral and Maxillofacial Radiology on selection criteria for the use of radiology in dental implantology with emphasis on cone beam computed tomography. Oral Surg Oral Med Oral Pathol Oral Radiol 2012;113: 817-826.

38. SEDENTEXCT Project. Chapter 4, Justi cation and referral criteria. Surgical applications. Implant dentistry In: Radiation Protection: Cone Beam CT for Dental and Maxillofacial Radiology. Evidence based guidelines 2011 (v2.0 Final). http://www.eadmfr.info/sites/ default/ les/ guidelines_nal.pdf. Accessed January 14, 2013.

39. Anil Kumar Nagarajappa, Neha Dwivedi and Rana Tiwari. Artifacts: The downturn of CBCT image. J Int Soc Prev Community Dent. 2015 Nov-Dec; 5(6): 440-445. 